\title{
Music Narrative Research on Shitang Yuejie Song*
}

\author{
Qunying Wang \\ School of Music \\ Shaoguan University \\ Shaoguan, China 512005
}

\author{
Guangtao Cao \\ School of Foreign Languages \\ Shaoguan University \\ Shaoguan, China 512005
}

\begin{abstract}
The Shitang Yuejie song is a unique style of north of Guangdong folk songs, mainly has two themes of labor theme and love. From the perspective of classic narrative, there are three narrative types: time narrative, spatial narrative, and character narrative. Throughout it is the female perspective. The Yuejie song is extensive, but the only constant is the unique female theme narrative. The female narrative is good at combining the situation with the virtual and the real. The Yuejie song uses the narrative of the story, and the sensation of the story makes the song of the month sister very lyrical, which causes the soul of the singer and the listener to resonate strongly.
\end{abstract}

Keywords-Shitang Yuejie song; female narrative; narrative song (Lili song); drama song

\section{INTRODUCTION}

Shitang Yuejie Song is popular in Shitang Town, Renhua County, a mountainous county in the northern part of Guangdong Province. The town has a total area of 80 square kilometers and a total population of over 13,000. The distribution area of Yuejie Song includes Shitang Village, Louxia Village, Tangxia Village, Huochong Village, Xinwu Village, Liyuan Village, Zaohetian Village and Caiwu Village. Compared with other folk song art, Shitang Yuejie Song has many unique artistic features. The lyrics of the Yuejie song are sung in the dialects unique to Shitang Village, and only the woman sings in the Yuejie song hall, and the men do not sing. The Yuejie song of the month do not sing, but only sing in the evening of the first lunar August 1st to August 15th, and do not sing during the day. The monthly sister song is only circulated in Shitang Town, Renhua County, which constitutes its unique regional characteristics. The Yuejie sister song belongs to the cappella, and there are solo, chorus, antiphonal singing, table singing and other singing forms. The Yuejie Song is a kind of folk song with distinctive moon god worship and original witchcraft features. It believes in the moon god and has a mysterious folk belief color. [1] In addition to the above characteristics, Shitang Yuejie songs has been circulated for thousands of years, and their charm is also in their distinctive

*Fund project: Social \&Science Planning of Shaoguan in 2016 (Research Base of Shao Culture). "Inheritance Rescue Record of heir of provincial non-material legacy Shitang Yuejie Song" (J2016012)

The research project "Live Collection and Research of Music Activity about Renhua Shitang Yuejie Song" of Shaoguan University in 2017 (SZ2017SK06) themes and unique narrative art.

\section{THE TwO MAJOR THEMES OF LABOR AND LOVE IN SHITANG YUEJIE SONG}

The theme of the Yuejie song is very extensive, involving a large number of agricultural production, life, solar terms, or narrative lyrics, or narrative seasonal, or describe the world's warm and warm world, or singing love. The lyrics content of Yuejie Song describes the social and economic forms, religious beliefs and cultural customs of Shitang Village. It is not only the traditional culture of women in Shitang Village, but also a beautiful folk song. It has unique nationality, life and regionality. It spreads the knowledge of production and life and the traditional culture of history, with a strong local flavor. Labor is the true nature of the working people, and Shitang Yuejie song is no exception. A lot of monthly sister songs are full of affirmation of labor and praise of laborers. Labor has brought about the development of human society, promoted the progress of human civilization, and has had an important impact on people's production and life. Therefore, the theme of labor has always been highly respected. Praise for labor and laborer reflects the importance attached to agriculture and the affirmation of the working people under the traditional farming civilization in northern Guangdong. Under the influence of farming civilization and Confucian ethics, Shitang women, who are diligent and kind, selfimprovement, love labor, praise labor, discover the beauty of nature in labor, flash industrious virtues in the harvest, and always maintain the true nature of the working people.

Love is the eternal theme of all art. The reason why Shitang Yuejie songs have been passed down through the ages is not only because of the colorfulness of the content or the twists and turns of the lyrics, but also because of its strong feminine spirit and profound cultural heritage, which embodies the aesthetic ideals and wishes of Shitang women at that time. Shitang Yuejie song also has a description of the love of talented and beautiful ladies, but there are only a few, only one or two, for example, the drama song "Xiucai Junzi". More is to reflect the love and happiness of the people's free and happy love and marriage and praise, such as "Eighteenyear-old Jiaojiao and three-year-old husband ", "Zhengyue brother to pick up the sister", "Yuanyeniang", "He Yanyan, Pengpeng Fei, "Shilun Sibie", "Yilun Sipian", "Jiuzuanli Baiyangyang", "Yipi Qingcai", "Gaomenkan Dimenlou", "Liu Sanniang", " YitiaoHegan", "Zhengyue brother to pick up the sister", "red paper flowers", "Yuanweixinv is 
Guanyin", "Taoqin song" and so on. The unique female narrative of Yuejie Song reflects the sisters' love and sincere love between Shitang women, and also meets the psychological needs they need to support each other.

\section{ThreE MAJOR TyPes OF THE YUEJIE SONG ClASSIC NARRATIVE}

The three basic elements of the classic narrative are arranged according to importance, followed by time, space and characters. According to this standard, Shitang Yuejie songs can be divided into character narratives, time narratives and spatial narratives.

\section{A. Time Narrative}

Shitang Yuejie songs in the passage of time, the lyrics usually use straightforward, paved and Bixing, and many other methods, it is often narrated by Changshier, Cahngshi, and Changwugeng, those are common in folk songs. Such as "Xiuxiangbao", "Shieryuehua", "Laocaicha", "Daochacha", "Zhuyege", "Jijiege", "Xincaicha", "Xiangbaoge", "Xiuxiangbao", "Zhengyue brother to pick up the sister", "Tongqiange", "Nongyege", "Wugeng Jizai", "Tanlangdiao", "Narrative song (Lili song)", "Haitangge", "Changgong Song", "ShiyueYingtai Xiuhua", "Tiaoyanggu", "Mupaige" and so on. These twelve paragraphs and five paragraphs of lyrics are presented in different levels in different months (January-December) or five-more (1-5), focusing on a theme, from the first month to the December, from the first Geng to the fifth Geng, each time space displacement is a turning or development of a plot, the music image is clear.

\section{B. Spatial Narrative}

Time and space research in classical narratology is regarded as an important factor in the composition of texts, mainly referring to the background and environment of events. In recent years, the emphasis on "spatial narratology" has met with literary anthropology and cultural studies. The object of spatial narratology is the imaginary space constructed by narrative text, which has all the attributes of real space."[2]

The central events reflected in the Yuejie songs are all in the space where the women in the villages, such as the boudoir, the courtyard, the pond, the stream, the market, the tea field, etc., are synchronized at the beginning and end of the event. "In the ancient Chinese narrative structure, space factors and other factors occupy a more important position than the West." [3] Every process of the month's sister song event is related to the transformation of space. Such songs include "Gengzaozao", "Wubeiyuanli (Tuanyuan Song)", "Ritouyichu Sijihua" and many Changshi and Changshier songs.

The language of the Yuejie songs is relatively straightforward, naturally expressing life and reflecting people's thoughts and feelings. Like "Changgong Song", it is a direct description of the work of men and women in August. The "Yuanyeniang" uses the meaning of the twists and turns, concealing the ideology of the women in Shitang. There was a sharp contradiction between the old women's wishes and the feudal marriage system, and the lyrics were clever and thought-provoking.

\section{Character Narrative}

1) Female character identification: In the lyrics of the Yuejie song, Shitang female is the protagonist, but at the same time it is a vulnerable group that is oppressed and suppressed. In the long feudal society, women were deeply subjected to various oppressions of monarchy, theocracy, tribes and husbands. The Yuejie Song is a stylized and open ceremony for women who live at the bottom of the society to use the sacredness and majesty of the Moon God to vent, express, express their inner sorrow and sorrow or good yearning, fighting these oppressions by refusing the participation of men.

2) Love life, love labor, pursue beauty, yearn for love: These appeals of Shitang women have been freely and vividly displayed in more than one hundred Shitang Yuejie songs collected, showing the interest and talent of Shitang people in the real production and life and love marriage materials.

\section{FEMAlE NARRATIVE AND FUNCTION OF SHITANG YUEJIE SONG}

The lyrics of the Yuejie song mainly involve the ceremony of the Yuejie song hall, the work of farming, the shortness of parents, the relationship between mother-in-law, love and marriage, historical knowledge, etc. According to the perspective of female narrative, the female narrative content of Shitang Yuejie song mainly has the following contents and functions:

Female moon god worship: It mainly describes typical customs and rituals such as "Jieyuejie", "Miyuejie" and "Songyuejie". Such songs are only sung during the ceremonies of the Moon Sisters, and they have certain religious beliefs and superstitions.

Women's Sufferings: Mainly showing the painful life of the working people in the old society, such as "Changgong Song", "Dangbing Song", "Mupaige", and "Fangniu Nenwa". "Changgong Song" profoundly expresses the old class contradictions, outlines the cruel exploitation of the peasants by the landlord class, reveals the grief and suffering of the peasants' inner life, and aims to arouse the resonance of the oppressed and inspire the people to fight against their destiny. "Changgong Song" because of its certain musical form can give people the role of adjustment and enthusiasm, causing people to resonate and sympathize. Singing such songs can not only vent the singer's own emotions, but also play a role in propaganda and education. It is a weapon for the social struggle and self-awakes of Shitang people in the old times, and has a positive self-education effect on the working people.

Female Marriage and Love: First of all, this kind of song is a love song, reflecting the true love and simple love of Shitang women, pursuing free love and happy life. For example, "Xiuxiangbao", "Dilang Song", "Tanlangdaio", "Wugenggu", "Peach Blossoms", "Shangling Kanzhu", "A 
Pot of Flowers", "New Buy Zhilan", " New Qiwawu, etc. "Xiuxiangbao" belongs to the palace style, the tune is clear, the melody is beautiful and moving, and the true love between men and women is truly expressed. In addition, there are many Yuejie songs that mainly show the painful life of Shitang women in the old days such as "He Yanyan, Pengpeng Fei", and so on. In the old days, the status of Shitang women was underground and was dominated by feudal regimes, ethnic rights, theocracy, and husbands. "Three cardinal guides and the five constant virtues", "the three obediences and the four virtues", "arranged marriage", "formal wedding" and other ethics only bind them, and there are many such subjects. Such as "Eighteen-year-old Jiaojiao and three-year-old husband ", "Zhengyue brother to pick up the sister", "Yuanyeniang", "He Yanyan, Pengpeng Fei, "Shilun Sibie", "Yilun Sipian", "Jiuzuanli Baiyangyang", "Yipi Qingcai", "Gaomenkan Dimenlou", "Liu Sanniang", " Yitiao Hegan", "Zhengyue brother to pick up the sister", "red paper flowers", "Yuanweixinv is Guanyin", "Taoqin song", "Xiucai Junzi", "Saobalai", and so on.[4] Complaints against the unreasonable marriage system of the old society, resentment of the unfair treatment of parents prefer boys to girls, revealing the coldness and abandonment of the bitterness at the home of the husband, reflecting the suffering and tragic fate of the old Shitang women. The idea of male superiority and female tempering forces women to be restricted. Only in the "Yuejie Song Hall" activity of MidAutumn Festival night, this restriction of women will be liberated, and then they will express their inner feelings and feel the joy of freedom. These lyrics not only express their desolation, but also express a resistance to inequality, which has certain historical value for promoting women's liberation.

The virtue of women: quite a part of the traditional ethical thoughts of our country existed in the Shitang Yuejie songs, and they were able to spread in the local generations with the help of the monthly sister songs. These songs use Confucian benevolence as the basic coordinates to persuade people to stay away from unethical behaviors such as laziness, gambling, alcoholism, deception, etc., reflecting the good and evil ideas and traditional virtues of Shitang people in real life, Such as "Shiquanlang", "Lazy Song", and so on."Education in interesting activities" gives the Yuejie Song a tense artistic vitality, such as "Wugeng Jizai" and "Siqiujie" have a strong self-entertainment. But even if it is very entertaining, it has its own content and social awareness, propagating certain opinions in entertainment, certain love and aesthetic views. The above two songs also reveal the embarrassment and optimism of the happy life of Yuejie. And "Dilongchuan" can spread certain knowledge in the fun, express people's love of life and freedom to give people education.

Women labors. "Jijiege", "Xincaicha", "Daochacha", "Zhuyege", "Nongyege", "Wubei Yuanli Zhongdouhua Sishuilangtou Sishuihua". These songs have a strong sense of life, describing the customs and customs of the northern Guangdong and the optimistic and simple character and style of the working people. They have the functions of propaganda, education and entertainment. Through such songs, you can get a wealth of regional production knowledge, life knowledge and etiquette knowledge.

Women's view of history: There is a song in the Yuejie song called "Lili Song", for example, "The Narrative Song", the lyrics directly tells many famous historical events and heroic deeds in the early Tang Dynasty. There is no storyline in the song, which is mainly used to popularize historical knowledge.

Women's daily life: such songs account for a large proportion of the Yuejie songs. Such as "Xiuxiangbao", "A comb", "Xiangbaoge", "Shiliu Dahua", "Xincaicha", "Shieryue Song", "Long and long", "Dilongchuan", "Reunion song", "One egg with two egg yolks", "Teapot song", "Sing Rural", "Zhima Yingqin", "Zhuye song", "Nine brothers with a little sister", "Pomegranate flowering" is of Zheng style, and it is the most representative song in "Miyue Jie". The melody is beautiful. Express people's yearning for a better life, eager to live like a pomegranate, the more blossoming and the more prosperous. The "Dilongchuan" is of $\mathrm{Yu}$ style. The lyrics of the "Dragon Boat" describe the lively scenes of people racing dragon boats. The tunes are simple and elegant, and the proper rest just sings people's excitement. Some of these songs express the daily life of Shitang people, full of vitality and rich life, reflecting the warm and innocent nature of Shitang people, the rhythm is bright, lively and lively, and has a strong entertainment. For thousands of years, rural cultural life has been lacking, and women in rural areas have become more prominent. They rely on editing and singing Yuejie songs to adjust their lives.

\section{CONCLUSION}

In summary, among the various narrative modes mentioned in the above different perspectives, the labor theme and the love theme are the core themes, and the female narrative is the most vivid narrative feature of Shitang's Yuejie song. Women tend to like euphemistic narrative patterns and are good at expressing. The Yuejie songs tend to adopt Bixing art techniques of the narrative and lyric, the combination of the virtual and the real, and adopt the two kinds of narrative techniques of "repetition" and "contrast", usually in the form of multi-section music songs that are repeatedly sung in a basic tune. The same is true of Shitang's Yuejie song. Such as "Reunion Song", the image is simple and contains multiple meanings. Shitang's Yuejie song is narratively, it is more lyrical, and mainly lyrical. The relationship between the "things" of the narrative in the Yuejie songs and the "lyric" of lyrics is considered to be narrative-based.

Feminine feelings are delicate and sensitive, good at sensation. For the Yuejie song, lyricism itself is also a narrative, a soft narrative. The lyrical Yuejie song is a Yuejie song that is created by the impression of real life and the emotional reaction at the same time. The "lyric" of the lyrical Yuejie song is mainly a feeling, a concept. Its performance and expression are often indirect and tortuous, the scene is integrated, inseparable, the lyric needs "things" support, and the narrative's intention is to write lyric. Therefore, the vast 
majority of the Yuejie songs can be classified into the lyric narrative of this kind of feeling and scene completely blended theory. The Yuejie songs make good use of Bixing imagery and contrast techniques. The emotional paving is often implicit in the plot narrative, such as "Reunion Song". The Yuejie songs are beautiful, comfortable, narrative, and lyrical. Most of the content is to sing the suffering of women in the old society and express their hope for future life. While lamenting their desolation, they also expressed their resistance in an angry manner, such as: "Eighteen-year-old Jiaojiao and three-year-old husband" and so on.

The Yuejie songs only have a few pure narrative songs. In terms of quantity, they can also show the characteristics of female narratives, that is, women do not pay much attention to history and current affairs. For the narrative Yuejie song, that is, the Yuejie song that is created by the emotions of the characters in the event or story, the narrative Yuejie song is based on lyricism, not from rural life scenes, but from the borrowing of traditional historical storytelling and novel stories. Its purpose is to spread historical knowledge and reflect some people's likes and dislikes or love concepts. The Yuejie songs with the narrative as the main style include "narrative songs", "Haitang songs", Drama songs "Xiucai Junzi", and the song "Liu Sanniang". The first two of them are the dissemination of historical knowledge. There is no storyline. The later songs are sung Liang Shanbo and Zhu Yingtai's love story and Liu Sanniang's unfortunate marriage. In "Xiucai Junzi", Liang Shanbo and Zhu Yingtai are typical talented scholars. Shitang people are willing to use their love stories to show their worship and sustenance. The tragic ending of Liang and Zhu's marriage in "Xiucai Junzi" not only reflects the resistance of Shitang women to feudal ethics, parents' arranged marriages, and sense of hierarchy, but also is their pursuit of free marriage and individuality.

\section{REFERENCES}

[1] Wang Qunying.Study on ShitangYuejie Songs [J].Journal of Shaoguan University, 2014(3): 39-42. (in Chinese)

[2] Chen Dezhi. Metaphor and Paradox: Space, Spatial Form and Space Narrative[J]. Jiangxi Social Sciences, 2009 (9): 63-67. (in Chinese)

[3] Luo Gang. Introduction to Narratology [M].Kunming: Yunnan People's Publishing House, 1994: 79. (in Chinese)

[4] Wang Qunying. The Artistic Features of "Yuejie Song" in Shitang Ancient Village, Renhua County, Northern Guangdong Province[J].Journal of Xinghai Conservatory of Music, 2014 (3):4551. (in Chinese) 